\title{
Hazardous Postoperative Outcomes of Unexpected COVID-19 Infected Patients: A Call for Global Consideration of Sampling All Asymptomatic Patients Before Surgical Treatment
}

\author{
Gauri Raman Gangakhedkar' ${ }^{1}$ (D) Sridhar Sundaram ${ }^{2} \cdot$ Mihir Raman Gangakhedkar $^{3} \cdot$ \\ Manali Prakash Shilotri ${ }^{3}$
}

Published online: 26 June 2020

(C) Société Internationale de Chirurgie 2020

We read with great interest the scientific review by Nahshon et al., titled 'Hazardous Postoperative Outcomes of Unexpected COVID-19 Infected Patients: A Call for Global Consideration of Sampling all Asymptomatic Patients Before Surgical Treatment' [1]. Universal testing for patients undergoing surgery promises to weed out those who slip through the diagnostic criteria in the pre-operative period. It would further allow the physicians to strike a balance between the urgency of surgery, allowing recover by deferring surgery, thus reducing mortality.

The article points to an inordinately high fatality in postoperative patients (14/64 or $21.8 \%$ ) in comparison with the $2-5 \%$ in the general population, indicating the need for increased vigilance among those undergoing surgical procedures in these testing times.

The authors conclude that patients must test for COVID19 before they undergo surgery using RT-PCR, the gold standard for testing for COVID-19 [2]. What must be considered is that RT-PCR has its own shortcomings as a testing modality. Depending on the site of sampling, the rate of swab positivity could range between 53.3 and $71 \%$ and that too, somewhere between 2 and 8 days after exposure [3]. This attests to the fact that merely testing negative for COVID-19, would not rule out the possibility of the patient being an asymptomatic carrier. It also remains vital that patients that do not have the disease, do not contract it, during the course of their stay in the

Gauri Raman Gangakhedkar

gauri2903@gmail.com

Seth Gordhandas Sunderdas Medical College, Mumbai, India

2 Seth GS Medical College and KEM Hospital, Mumbai, India

3 All India Institute of Medical Sciences - Rishikesh, Rishikesh, India hospital. With both these possibilities in mind, even if patients test negative for COVID-19, it is of great import that they be presumed to be infected, and measures such as use of appropriate personal protective equipment, social distancing, and meticulous respiratory hygiene be implemented to protect both the healthcare providers and other patients in the hospital.

An important question that arises with regard to the clinical course of the patients is that due to the variability in the diagnostic tests, it becomes impossible to point out if the 51/64 patients, who tested positive in the post-operative period, were infected before the hospital admission or during the course of their hospital stay.

It also seems worth looking into, that of the vast volumes of the literature available regarding COVID-19, and with over 6 million people being affected with it worldwide as of 30 of May, the peri-operative outcomes from these 64 patients might not reflect the global reality.

That being said, the opportunity to diagnose and possibly reduce mortality among those who do test positive during a pre-operative screening, cannot be dismissed, particularly in areas with high prevalence rates for COVID19.

This, however, will only be possible when we have at our disposal improved testing modalities that reduce the window from the time of infection to detection, allow more uniform rates of detection and possess increased sensitivity to detect the virus, without compromising the specificity of the same, preventive strategies seem to be a better alternative to universal testing, in our endeavours to protect our healthcare workers. 


\section{References}

1. Nahshon C, Bitterman A, Haddad R, Hazzan D, Lavie O (2020) Hazardous postoperative outcomes of unexpected COVID-19 infected patients: a call for global consideration of sampling all asymptomatic patients before surgical treatment. World J Surg. https://doi.org/10.1007/s00268-020-05575-2

2. Tahamtan A, Ardebili A (2020) Real-time RT-PCR in COVID-19 detection: issues affecting the results. Expert Rev Mol Diagn. https://doi.org/10.1080/14737159.2020.1757437
3. Zhai P, Ding Y, Wu X, Long J, Zhong Y, Li Y (2020) The epidemiology, diagnosis and treatment of COVID-19. Int $\mathrm{J}$ Antimicrob Agents. https://doi.org/10.1016/j.ijantimicag.2020. 105955

Publisher's Note Springer Nature remains neutral with regard to jurisdictional claims in published maps and institutional affiliations. 